B. M. Shields $\cdot$ B. Knight $\cdot$ M. Turner •

B. Wilkins-Wall · L. Shakespeare · R. J. Powell •

M. Hannemann · P. M. Clark · C. S. Yajnik •

A. T. Hattersley

\title{
Paternal insulin resistance and its association with umbilical cord insulin concentrations
}

Received: 17 August 2005 / Accepted: 3 April 2006 / Published online: 16 May 2006

C) Springer-Verlag 2006

\begin{abstract}
Aims/hypothesis: Fetal growth is influenced by genetic factors as well as the intra-uterine environment. We hypothesised that some genetic factors may alter fetal insulin secretion and insulin action. Subjects, materials and methods: To assess this, we analysed plasma insulin concentration in umbilical cord blood from 644 normal, term, UK Caucasian deliveries from the Exeter Family Study of Childhood Health. We tested for associations between cord insulin and each of parental anthropometry, fasting glucose, insulin and lipids. Results: As expected, cord insulin concentrations correlated with all measures of birth size (weight, length, head and arm circumferences, sum of skinfold thicknesses, ponderal index: $r=0.16-0.4, p<0.01$ for all) and maternal BMI $(r=0.11, p=0.005)$, maternal glucose $(r=0.25, p<0.001)$ and maternal insulin resistance $(r=0.23$, $p<0.001)$. Paternal fasting insulin and insulin resistance were correlated with cord insulin $(r=0.15, p=0.006 ; r=0.13$, $p=0.001$, respectively), and this was independent of paternal
\end{abstract}

B. M. Shields · B. Knight · A. T. Hattersley $(\bowtie)$

Peninsula Medical School,

Barrack Road,

Exeter, EX2 5DW, UK

e-mail: A.T.Hattersley@ex.ac.uk

Tel.: +44-1392-406806

Fax: +44-1392-406767

B. Knight · M. Turner · B. Wilkins-Wall · M. Hannemann

Heavitree Hospital,

Royal Devon and Exeter NHS Foundation Trust,

Exeter, UK

L. Shakespeare · P. M. Clark

The Regional Endocrine Laboratory,

University Hospital Birmingham NHS Trust,

Birmingham, UK

R. J. Powell

Research and Development Support Unit,

Royal Devon and Exeter NHS Foundation Trust,

Exeter, UK

C. S. Yajnik

King Edwards Memorial Hospital and Research Centre,

Pune, India
BMI. Multiple linear regression analysis revealed paternal insulin resistance to be a predictor of cord insulin concentrations, independently of maternal factors. Conclusion: Our results show an independent relationship between paternal insulin resistance and cord insulin concentrations. This is consistent with heritability of insulin resistance from father to offspring and a compensatory increase in fetal insulin secretion, the latter occurring prenatally before the homeostatic feedback loop between glucose and insulin is established.

Keywords Fetal · Foetal · Growth · Insulin · Insulin resistance $\cdot$ Paternal $\cdot$ Umbilical cord

Abbreviations EFSOCH: Exeter Family Study of Childhood Health · HOMA-R: homeostasis model assessment of insulin resistance

\section{Introduction}

Fetal growth is regulated by the intra-uterine environment and genetic factors. Relationships between fetal growth and environmental factors such as maternal glucose, maternal size, parity, and smoking are well established $[1,2]$ and there is evidence for genetic regulation of fetal growth from studies on sex-related differences, racial differences and associations with paternal height, [1].

Insulin-mediated growth is a major pathway in fetal growth regulated by the intra-uterine environment [3]. This is most clearly demonstrated by the macrosomia associated with diabetic pregnancies. Pedersen [4] proposed that increased fetal growth in diabetic pregnancies is due to increased insulin secretion by the fetus in response to exposure to a high maternal glucose concentration. Birthweight is directly correlated with maternal glycaemia, both fasting and stimulated glucose levels, in the non-diabetic pregnancy [5]. Other environmental factors altering fetal insulin concentrations and growth include maternal insulin sensitivity during pregnancy [6-8] and maternal size [9], with associations also seen with maternal nutrition [10], parity [10,11], and social class [10]. 
There is also growing evidence of a role for genetic regulation of insulin-mediated growth. Severe fetal mutations that greatly alter insulin secretion, or insulin action, such as those associated with pancreatic agenesis or mutations in the glucokinase gene leading to MODY, considerably alter birthweight [12-14]. Although these mutations are rare, they have established the principle that fetal genes can alter insulin-mediated growth.

We hypothesised that genetic factors are important in the regulation of insulin-mediated growth in normal pregnancies. There is limited evidence of genetic determinants of fetal insulin concentrations from studies on twins [15] and different ethnic groups [16-18], and it has been suggested that that there may be sex-related differences, as evidenced both by the finding that girls are intrinsically more insulinresistant than boys in early childhood [19] and by the sexual dimorphism seen in the insulin-like growth factor axis present at birth [20]. Recent studies have also noted an association between low birthweight and diabetes and insulin resistance in parents and grandparents [21-25], and it may be that this relationship is a result of inherited insulin resistance altering insulin-mediated growth as proposed by the 'fetal insulin hypothesis' [14].

To date, no studies have looked at the relationship between paternal factors and cord insulin. A relationship between umbilical cord insulin and paternal measures (independent of maternal measures) would provide further support for a role of genetics in the regulation of fetal insulin concentrations. In this study, we show that there is a direct association between paternal insulin resistance and cord insulin concentrations, and that this association is independent of the intra-uterine environment.

\section{Subjects, materials and methods}

Subject recruitment and laboratory analyses

Families were recruited as part of the Exeter Family Study of Childhood Health (EFSOCH), a 5-year prospective study, started in 1999, with the aim of examining genetic influences on fetal and early growth. Detailed anthropometric measurements and fasting glucose, insulin and lipid concentrations were measured in both parents at 28 weeks gestation. Similar anthropometric measurements were taken from their child at birth. This study has been described in detail previously [26].

Cord blood was obtained from the placental end of the cord after the placenta was delivered, and centrifuged as soon as possible. Cord plasma insulin and fasting insulin in both parents were measured using an immunochemiluminometric assay (Molecular Light Technology, Cardiff, UK). The assay is specific for insulin and the interassay coefficients of variation were less than $9.0 \%$ over the concentration range reported.

Socio-economic status was assigned by Townsend scores based on enumeration districts by post code [27]. A Townsend score of 0 indicates the average for the UK, with positive scores indicating more deprivation, and negative scores representing more affluence.

Insulin resistance was calculated in both parents from fasting glucose and insulin using the homeostasis model assessment of insulin resistance (HOMA-R) program for specific insulin measurement kindly provided by Levy (OCDEM, Churchill Hospital, Oxford, UK) [28, 29].

Ethical approval was given by the North and East Devon Local Research Ethics Committee and informed consent was obtained from the parents of the newborns.

\section{Statistics}

Data are presented as means and standard deviations. All data were tested for normal distribution and log transformed where necessary. Geometric means are therefore presented for these variables. Relationships between cord insulin and birth measurements were estimated using partial correlations adjusting for gestational age, and sex when combining boys 'and girls' measurements. Differences in cord insulin concentrations between boys and girls were assessed using the Student's $t$-test. Pearson correlation coefficients were used to estimate the relationships
Fig. 1 Flow chart showing selection of parents and babies in EFSOCH

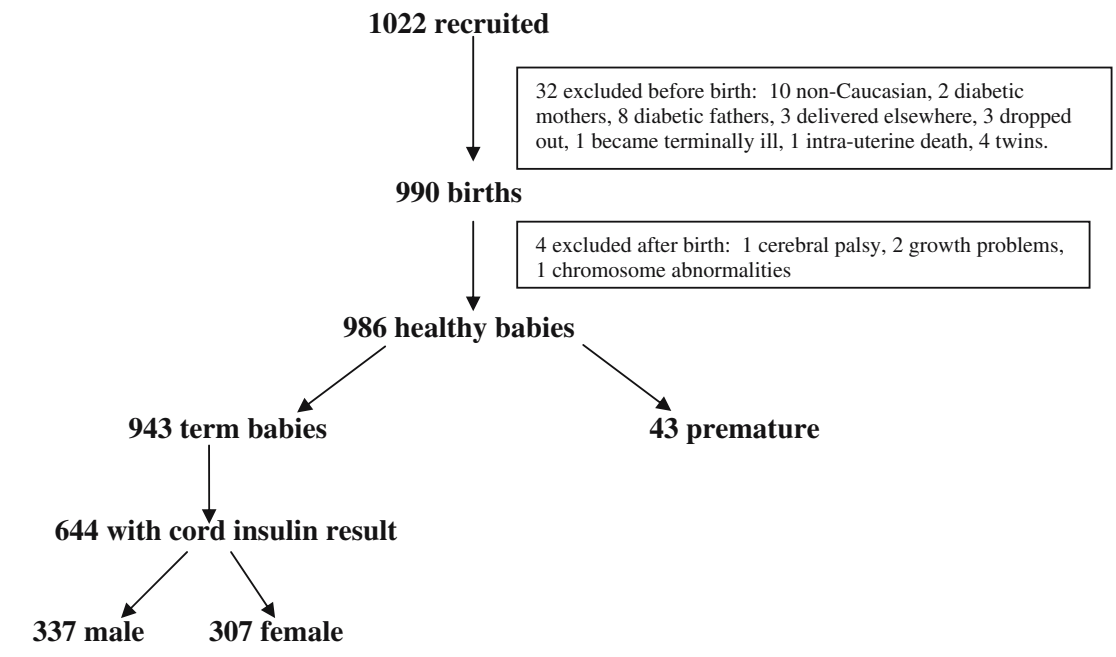


between parental variables and cord insulin concentrations. Multiple linear regression analysis was used to assess the independent relationship of paternal insulin resistance with cord insulin concentrations when accounting for potential confounders of maternal glucose, socio-economic status, maternal smoking, maternal insulin resistance and parental age. Further exploration of the relationship between paternal insulin resistance and cord insulin concentrations was conducted by using a similar pair-matching analysis to that carried out in a previous study [17] comparing Indian and Caucasian babies. Thus cord insulin concentrations in babies born to fathers in the highest tertile of insulin resistance were compared with those born to fathers in the lowest tertile of insulin resistance after pair-matching offspring for birthweight (within $100 \mathrm{~g}$ ) and gestation (within 5 days).

\section{Results}

Study cohort

Figure 1 details how families were selected for analysis. We present results on the 644 full-term babies from whom a cord insulin measurement is available. Those for whom a cord insulin sample was available were slightly heavier than those without (3,531 vs. $3,451 \mathrm{~g}, p=0.015)$, although there was no difference in gestation.

Characteristics of mother, father and baby are listed in Table 1 (birth measurements have been corrected for gestation at 40 weeks using the British 1990 Growth reference data [30]). The median Townsend score for families in EFSOCH was -0.24 (range: -6.61 to 8.85 ). A total of $288(45 \%)$ were primiparous pregnancies. Eighty-nine mothers (14\%) and 180 fathers $(28 \%)$ admitted to smoking in pregnancy. Birth was by normal vaginal delivery (454 babies), assisted delivery (84 babies), and Caesarean section (104 deliveries, 49 of which were emergency and 55 elective).

\section{Cord insulin measurements}

The median cord plasma insulin concentration was $37.7 \mathrm{pmol} / \mathrm{l}$. Girls had higher cord insulin concentrations than boys but this difference was not significant (geometric means: 41.0 vs. 37.4 pmol/l, $p=0.096$ ). Babies born by Caesarean section had significantly higher cord insulin concentrations than babies born by vaginal delivery $(56.7$ vs. $36.4 \mathrm{pmol} / 1, p<0.001)$. These babies were also heavier $(3,707$ vs. $3,555 \mathrm{~g}, p=0.001)$ and longer ( 50.9 vs. $50.5 \mathrm{~cm}$, $p=0.030$ ), when correcting for gestational age and sex.

Cord insulin concentrations with birth measurements

All cord insulin concentrations were corrected for gestation and sex, and in addition, boys and girls were analysed separately. As expected, cord insulin was positively correlated with all birth measurements in boys and in girls $(r=0.16-0.4, p<0.01$ for all) (Table 2$)$. The strength of relationships between cord insulin and birth size was similar in boys and girls and also similar for different birth measurements.

Parental measurements and cord insulin concentrations

\section{Maternal variables}

In keeping with previous studies, maternal 28-week fasting glucose, HDL-cholesterol and insulin concentrations, and

Table 1 Characteristics of mother and father at 28 weeks gestation and of babies at birth in 644 families recruited as part of the Exeter Family Study of Childhood Health

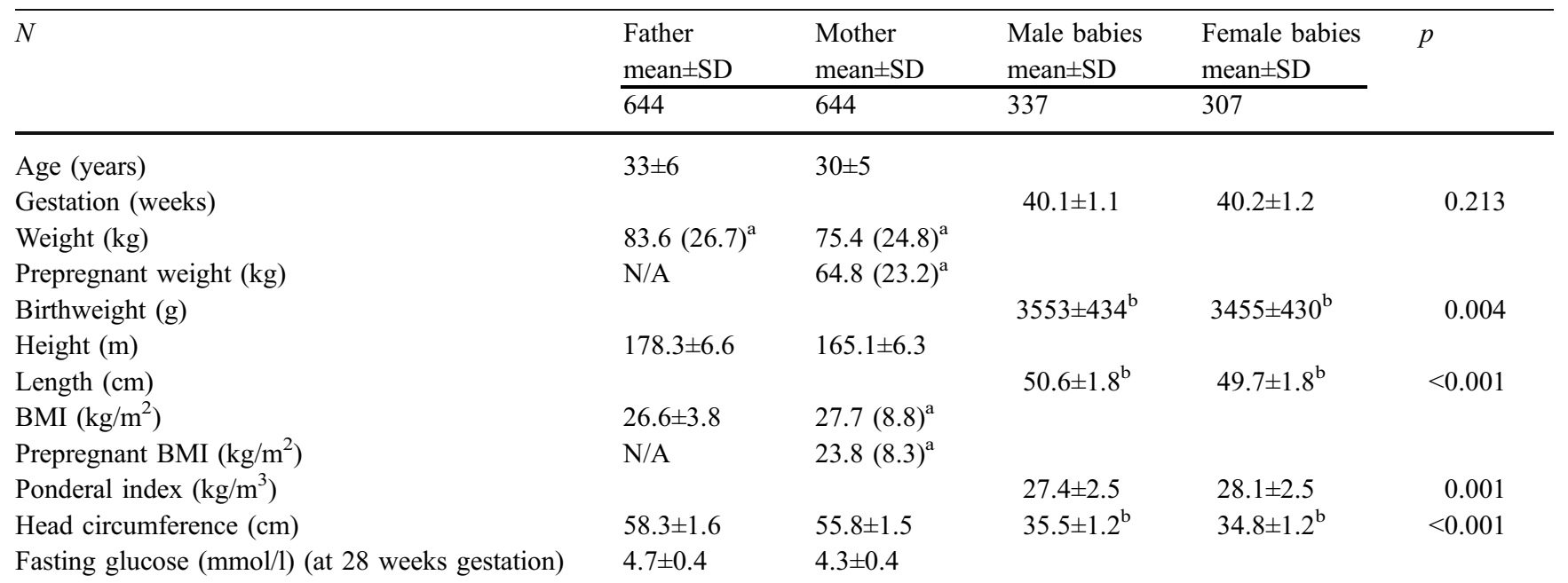

${ }^{\mathrm{a}} \mathrm{Log}$ transformed data-geometric mean (SD range) reported

${ }^{b}$ Corrected for gestation at 40 weeks using the British 1990 Growth reference data [30] 
Table 2 Correlations of cord insulin concentrations with birth measurements

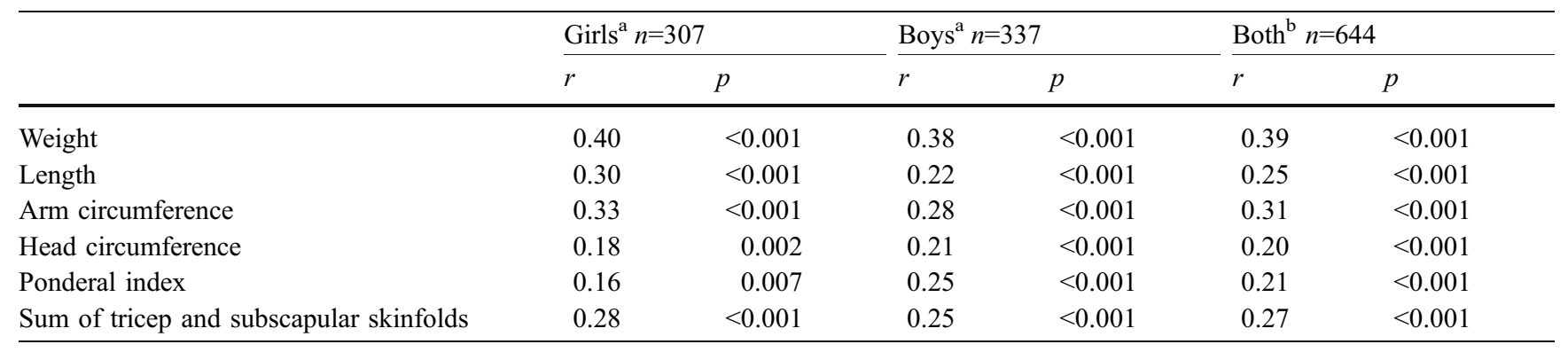

${ }^{\text {a}}$ Pearson correlation coefficients, adjusted for gestational age

${ }^{\mathrm{b}}$ Pearson correlation coefficients, adjusted for gestational age and sex of the baby

HOMA-R insulin resistance were all directly correlated with cord insulin concentrations (Table 3). Cord insulin concentrations were also significantly related to maternal age, 28-week weight, BMI and sum of skinfold thicknesses. Parity was associated with cord insulin concentrations, with primiparae having lower cord insulin concentrations than multiparae (36.5 vs. $41.2 \mathrm{pmol} / \mathrm{l}$, respectively, $p=0.035$ ). Socio-economic status and gestation did not significantly alter cord insulin concentrations.

\section{Paternal variables}

The relationships with paternal variables are shown in Table 3. Paternal HOMA-R insulin resistance and fasting insulin concentrations were positively correlated with cord insulin concentrations $(r=0.14$ and 0.15 , respectively, $p<0.001$ for both), and HDL cholesterol was negatively related $(r=-0.11, p=0.008)$. The relationships remained significant when correcting for maternal insulin resistance (Table 3). Correcting for paternal BMI made little difference to the correlation with paternal HOMA-R insulin resistance $(r=0.13, p=0.001)$. No non-significant relationship was changed by correction for maternal insulin resistance.

Inter-relationships between parental measurements, cord insulin and birth weight

For all parental variables that were significantly correlated with cord insulin concentrations, we tested for associations with birthweight. All maternal variables associated with cord insulin were significantly correlated with birthweight $(r=0.09-0.33$ for all, except HDL cholesterol, which had a negative correlation $r=-0.10 ; p<0.001$ to 0.018 for all). Measures of paternal anthropometry (head circumference and sum of skinfolds) were also related to birthweight $(r=0.14$ and 0.10 , respectively, $p<0.001$ and 0.009 ). However, measures of paternal biochemistry were not (paternal insulin resistance $[r=0.01, p=0.83]$, paternal insulin $[r=0.02, p=0.61]$ and paternal HDL cholesterol $[r=-0.04, p=0.38])$.

Table 3 Correlations of cord insulin concentrations with parental variables for 644 families (complete measurements not available on all parents)

\begin{tabular}{|c|c|c|c|c|c|c|}
\hline & \multicolumn{2}{|c|}{ Maternal variables } & \multicolumn{2}{|c|}{ Paternal variables } & \multicolumn{2}{|c|}{ (Corrected for maternal variable) } \\
\hline & $r$ & $p$ & $r$ & $p$ & $r$ & $p$ \\
\hline Age & 0.15 & $<0.001$ & 0.10 & 0.015 & 0 & 0.904 \\
\hline \multicolumn{7}{|l|}{ Anthropometry } \\
\hline Weight & 0.11 & $<0.001$ & 0.05 & 0.251 & & \\
\hline Height & 0.01 & 0.816 & -0.01 & 0.837 & & \\
\hline BMI & 0.11 & 0.005 & 0.05 & 0.175 & & \\
\hline Head circumference & 0.07 & 0.068 & 0.10 & 0.014 & 0.09 & 0.018 \\
\hline Sum of skinfolds & 0.14 & $<0.001$ & 0.12 & 0.003 & 0.10 & 0.015 \\
\hline \multicolumn{7}{|l|}{ Biochemistry } \\
\hline Triglycerides & 0.06 & 0.108 & 0.07 & 0.075 & & \\
\hline HDL-cholesterol & -0.12 & 0.004 & -0.11 & 0.008 & -0.08 & 0.038 \\
\hline LDL-cholesterol & 0 & 0.956 & 0.03 & 0.419 & & \\
\hline Fasting glucose & 0.25 & $<0.001$ & 0.02 & 0.666 & & \\
\hline Log fasting insulin & 0.22 & $<0.001$ & 0.15 & $<0.001$ & 0.12 & 0.003 \\
\hline Log HOMA-R & 0.23 & $<0.001$ & 0.14 & $<0.001$ & 0.11 & 0.006 \\
\hline
\end{tabular}

Pearson correlation coefficients, adjusted for gestational age and sex of the baby 
Multiple regression analysis of cord plasma insulin concentrations was carried out to assess the independent determinants of cord insulin concentrations (Table 4). Independent variables added were gestation and sex, measures of the intra-uterine environment (maternal glucose, smoking), and measures of insulin resistance with potential confounders (maternal HOMA-R insulin resistance, maternal BMI, maternal age, paternal HOMA-R insulin resistance, and paternal BMI). Paternal age was not added to the model due to its high correlation with maternal age $(r=0.66)$, which would have led to problems with multicolinearity. Data were assessed for model fit and there was no evidence of any departure from the model assumptions. Paternal insulin resistance was a determinant of cord insulin concentrations, independently of maternal factors and paternal BMI. Baby's sex was also a weak, but significant, independent predictor of cord insulin. As expected, maternal glucose and maternal insulin resistance were the strongest predictors of cord insulin.

To further explore the relationship between paternal insulin resistance and cord insulin concentrations, babies were split by tertiles of paternal insulin resistance (high, medium and low). The babies in the highest tertile of paternal insulin resistance were pair-matched for sex, birthweight (to nearest $100 \mathrm{~g}$ ) and gestation (to 5 days) with babies in the lowest tertile of paternal insulin resistance. On these criteria, it was possible to match 136 pairs. Babies born to fathers in the lowest tertile of insulin resistance had lower cord insulin concentrations than babies born to fathers in the highest tertile of insulin resistance (geometric means: 36.1 vs. $42.7 \mathrm{pmol} / 1$, respectively, $p=0.036$ ). This difference became greater when comparing lowest and highest tertiles of paternal insulin resistance corrected for paternal BMI (to obtain an estimate of 'intrinsic' insulin resistance) (geometric means: 33.5 vs. $43.1 \mathrm{pmol} / 1$ respectively, $p=0.005$ ).

Finally, we looked at the combined effect of both parents' insulin resistance on cord insulin concentrations (see Fig. 2). Maternal insulin resistance was also split into tertiles. Except for in the highest tertile of maternal insulin resistance, there was a trend for cord insulin concentrations to decrease from the highest to the lowest tertile of both maternal and paternal insulin resistance, although these trends did not quite reach significance ( $p>0.059$ for all). Babies with both parents in the highest tertile of insulin resistance had significantly higher cord insulin concentrations than babies with both parents in the lowest tertile of insulin resistance (geometric means: 49.3 vs. $31.1 \mathrm{pmol} / 1$, respectively, $p<0.001)$. A similar result was obtained when using tertiles of parental insulin resistance corrected for parental BMI (geometric means: 43.8 vs. 30.7 pmol/l, $p=0.003)$.

\section{Discussion}

Our study has demonstrated relationships of cord insulin concentrations with parental insulin resistance. The correlation with paternal insulin resistance, which is independent of the intra-uterine environment, is a novel finding and suggests there may be a genetic component to the regulation of cord insulin concentrations. The relationship is weak but robust, and remains when correcting for potential maternal confounders (Table 3); it is also

Table 4 Multiple linear regression analysis with $\log$ cord insulin as dependent variable

\begin{tabular}{llrrrrrr}
\hline & \multicolumn{1}{c}{$B$} & SE (B) & Lower 95\% CI & Upper 95\% CI & $t$ & $p$ & 0.015 \\
\hline 1 & & 0.058 & 0.024 & 0.011 & 0.104 & 2.437 & 0.095 \\
& Sex & 0.004 & 0.010 & -0.016 & 0.024 & 0.404 & 0.686 \\
Gestation & 0.179 & 0.033 & 0.114 & 0.243 & 5.438 & $<0.001$ \\
Maternal glucose & 0.147 & 0.182 & -0.212 & 0.505 & 0.804 & 0.422 \\
Maternal BMI & -0.047 & 0.024 & -0.094 & -0.001 & -1.987 & 0.047 \\
Parity (Primip) & -0.068 & 0.034 & -0.135 & 0.000 & -1.975 & 0.049 \\
Smoking & 0.154 & 0.050 & 0.057 & 0.252 & 3.109 & 0.002 \\
Log paternal IR & 0.055 & 0.024 & 0.009 & 0.102 & 2.334 & 0.020 \\
Sex & 0.005 & 0.010 & -0.015 & 0.025 & 0.528 & 0.597 \\
Gestation & 0.132 & 0.035 & 0.063 & 0.201 & 3.756 & $<0.001$ \\
Maternal glucose & -0.137 & 0.209 & -0.547 & 0.273 & -0.655 & 0.513 \\
Maternal BMI & -0.035 & 0.025 & 0.083 & 0.013 & -1.429 & 0.154 \\
Parity (Primip) & -0.048 & 0.035 & -0.117 & 0.021 & -1.365 & 0.173 \\
Smoking & 0.157 & 0.057 & 0.044 & 0.270 & 2.733 & 0.006 \\
Log paternal IR & 0.007 & 0.003 & 0.002 & 0.012 & 2.790 & 0.005 \\
Maternal age & 0.242 & 0.074 & 0.097 & 0.387 & 3.287 & 0.001 \\
Log maternal IR & -0.003 & 0.004 & -0.010 & 0.004 & -0.891 & 0.373 \\
Paternal BMI & &
\end{tabular}

Two models are presented: Model 1 includes sex, gestation, maternal BMI, maternal glucose, maternal smoking and paternal insulin resistance to assess the effect of paternal insulin resistance independently of the intra-uterine environment. Model 2 adds potential confounders such as maternal insulin resistance, maternal age and paternal BMI

$B$ Regression coefficient, $S E(B)$ standard error for $B, t$ the $t$-statistic defined by $B / \mathrm{SD}, R^{2}$ the coefficient of determination, $I R$ insulin resistance 
Fig. 2 The interactions of cord insulin concentrations and parental insulin resistance (in tertiles). The geometric mean of cord insulin concentrations according to maternal insulin resistance tertile is shown as rows and the geometric mean of cord insulin concentrations according to paternal insulin resistance tertile is shown as columns

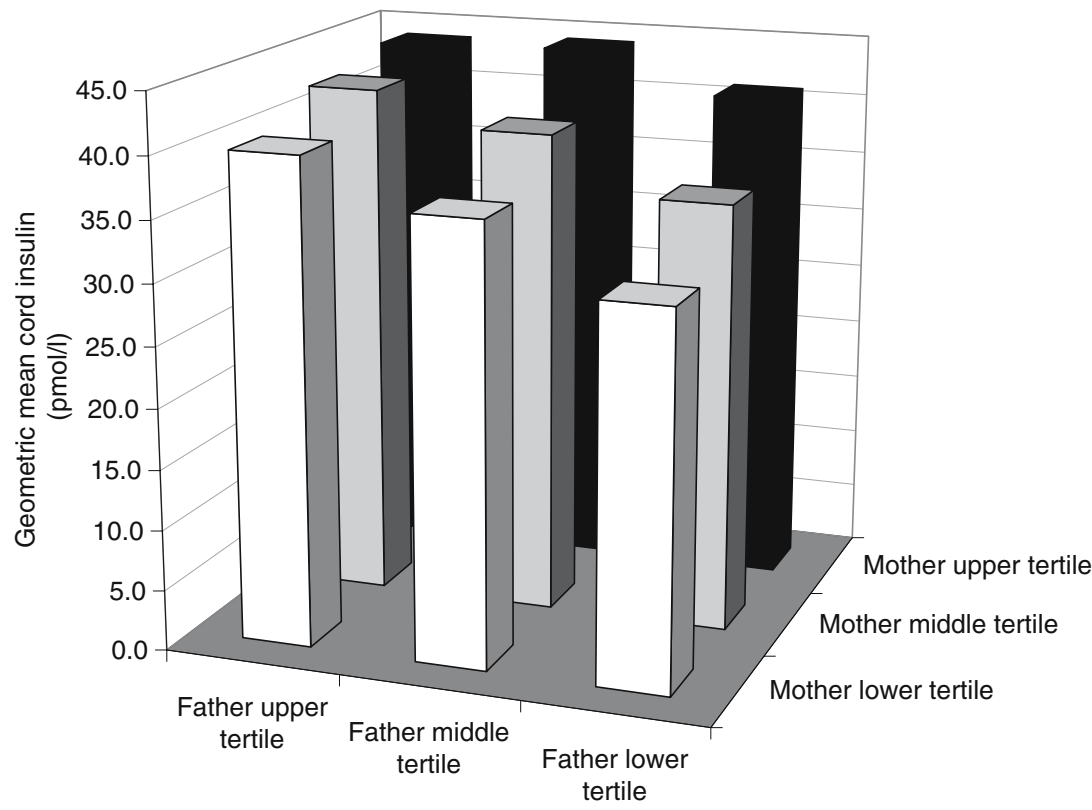

independent in a multiple linear regression analysis (Table 4) and is further confirmed by pair-matching analyses.

We have confirmed previous findings of associations between cord insulin concentrations and fetal growth [11, $17,31]$, although these studies did not find correlations with measures of skeletal size such as length. We are uncertain why the results of this study differ from others, but it may reflect the relatively large numbers in our study, the accurate prospectively collected data using a research protocol to measure length, the mode of collection of cord samples, and the use of a specific insulin assay. As predicted, we found correlations with measures of the intrauterine environment such as maternal glycaemia, maternal insulin resistance and maternal BMI, and relationships of these factors with both cord insulin and fetal growth have been reported previously [5-9]. These expected findings provide validation for our insulin results.

Paternal insulin resistance is an independent predictor of cord insulin concentrations that explains further variance in cord insulin in addition to already known determinants. Unlike the maternal parameters associated with raised cord insulin concentrations, paternal insulin resistance was not associated with increased fetal growth. This suggests heritability of insulin resistance from father to offspring, and the existence, pre-natally, of a compensatory increase in fetal insulin secretion, before the homeostatic feedback loop between glucose and insulin is established. The mechanism whereby this feedback occurs is not known. Indian subjects are more insulin-resistant than European Caucasians and a similar compensatory increase in cord insulin for genetic insulin resistance is seen in Indian babies, who have a higher cord insulin per similar birthweight than UK babies [17]. In this study we did not see the inverse relationship between paternal insulin resistance and offspring birthweight predicted by the 'fetal insulin hypothesis' [14]. This needs to be assessed on the whole dataset according to the published protocol [26].
We performed pair-matching analysis of birthweights to assess the effect of paternal insulin resistance on cord insulin concentrations in babies with similar birthweights. The higher insulin concentrations, despite similar birthweights, in babies born to the most insulin-resistant fathers compared with those born to the least insulin-resistant fathers further suggest that the relationship reflects differences in insulin resistance rather than insulin secretion. Hyperinsulinaemia may occur without evidence of insulin resistance, as determined by euglycaemic clamp, in populations known to be at high risk of insulin resistance such as patients with polycystic ovary syndrome [32], juvenile obesity [33] and the Pima Indian population [34]. So although hyperinsulinaemia is consistent with insulin resistance this cannot be assumed to be the cause.

Our study has limitations. The study design requires a participating father, leading to a selected cohort of twoparent families and so cannot be considered representative of all births. The measurement of cord insulin concentrations is an indirect measure of fetal insulin concentrations. Cord insulin measurements have been shown to be susceptible to the stress of delivery [31, 35, 36], which may add variability to the data. However, this may reflect the larger birthweights of babies requiring Caesarean section, as seen in our data. The relationships seen in our study remained, whether babies born by Caesarean section were included in or excluded from the analysis.

In summary, we have demonstrated that paternal insulin resistance is associated with umbilical cord insulin concentrations and that this effect is independent of maternal factors. This is consistent with the notion that there is genetic regulation of cord insulin concentrations, with insulin resistance being inherited by the fetus from their father. The mechanism for a compensatory increase in fetal insulin secretion occurring pre-natally, before the homeostatic feedback loop between glucose and insulin is established, is unknown and an important area for future study. 
Acknowledgements This study was funded by South West NHS Research and Development, Exeter NHS Research and Development and the Darlington Trust. A. T. Hattersley is a Wellcome Trust Research Leave fellow. B. Knight holds an NHS Research and Development studentship. The support of University Hospital Birmingham Charities is gratefully acknowledged (L. Shakespeare, P. M. Clark). This paper is dedicated to the memory of Tina Turner, who was a valued member of the Exeter Family Study team.

\section{References}

1. Kramer MS (1987) Determinants of low birth weight: methodological assessment and meta-analysis. Bull World Health Organ 65:663-737

2. Catalano PM, Kirwan JP (2001) Maternal factors that determine neonatal size and body fat. Curr Diab Rep 1:71-77

3. Fowden AL (1992) The role of insulin in fetal growth. Early Hum Dev 29:177-181

4. Pedersen J (1977) The pregnant diabetic and her newborn. Problems and management. Williams and Wilkins, Baltimore

5. Breschi MC, Seghieri G, Bartolomei G, Gironi A, Baldi S, Ferrannini E (1993) Relation of birthweight to maternal plasma glucose and insulin concentrations during normal pregnancy. Diabetologia 36:1315-1321

6. Catalano PM, Drago NM, Amini SB (1995) Maternal carbohydrate metabolism and its relationship to fetal growth and body composition. Am J Obstet Gynecol 172:1464-1470

7. Bernstein IM, Goran MI, Copeland KC (1997) Maternal insulin sensitivity and cord blood peptides: relationships to neonatal size at birth. Obstet Gynecol 90:780-783

8. Caruso A, Paradisi G, Ferrazzani S, Lucchese A, Moretti S, Fulghesu AM (1998) Effect of maternal carbohydrate metabolism on fetal growth. Obstet Gynecol 92:8-12

9. Soltani KH, Bruce C, Fraser RB (1999) Observational study of maternal anthropometry and fetal insulin. Arch Dis Child Fetal Neonatal Ed 81:F122-F124

10. Godfrey KM, Robinson S, Hales CN, Barker DJ, Osmond C, Taylor KP (1996) Nutrition in pregnancy and the concentrations of proinsulin, 32-33 split proinsulin, insulin, and Cpeptide in cord plasma. Diabet Med 13:868-873

11. Ong K, Kratzsch J, Kiess W, Costello M, Scott C, Dunger D (2000) Size at birth and cord blood levels of insulin, insulin-like growth factor I (IGF-I), IGF-II, IGF-binding protein-1 (IGFBP1), IGFBP-3, and the soluble IGF-II/mannose-6-phosphate receptor in term human infants. The ALSPAC Study Team. Avon Longitudinal Study of Pregnancy and Childhood. J Clin Endocrinol Metab 85:4266-4269

12. Hattersley AT, Beards F, Ballantyne E, Appleton M, Harvey R, Ellard S (1998) Mutations in the glucokinase gene of the fetus result in reduced birth weight. Nat Genet 19:268-270

13. Stoffers DA, Zinkin NT, Stanojevic V, Clarke WL, Habener JF (1997) Pancreatic agenesis attributable to a single nucleotide deletion in the human IPF1 gene coding sequence. Nat Genet 15:106-110

14. Hattersley AT, Tooke JE (1999) The fetal insulin hypothesis: an alternative explanation of the association of low birthweight with diabetes and vascular disease. Lancet 353:1789-1792

15. Verhaeghe J, Loos R, Vlietinck R, Herck EV, van Bree R, Schutter AM (1996) C-peptide, insulin-like growth factors I and II, and insulin-like growth factor binding protein-1 in cord serum of twins: genetic versus environmental regulation. Am J Obstet Gynecol 175:1180-1188

16. Simmons D (1994) Differences in umbilical cord insulin and birth weight in non-diabetic pregnancies of women from different ethnic groups in New Zealand. Diabetologia 37:930-936

17. Yajnik CS, Lubree HG, Rege SS et al (2002) Adiposity and hyperinsulinemia in Indians are present at birth. J Clin Endocrinol Metab 87:5575-5580
18. Delvaux T, Buekens P, Thoumsin H, Dramaix M, Collette J (2003) Cord C-peptide and insulin-like growth factor-I, birth weight, and placenta weight among North African and Belgian neonates. Am J Obstet Gynecol 189:1779-1784

19. Murphy MJ, Metcalf BS, Voss LD et al (2004) Girls at five are intrinsically more insulin resistant than boys: the programming hypotheses revisited-The EarlyBird Study (EarlyBird 6). Pediatrics 113:82-86

20. Geary MP, Pringle PJ, Rodeck CH, Kingdom JC, Hindmarsh PC (2003) Sexual dimorphism in the growth hormone and insulin-like growth factor axis at birth. J Clin Endocrinol Metab 88:3708-3714

21. Lindsay RS, Dabelea D, Roumain J, Hanson RL, Bennett PH, Knowler WC (2000) Type 2 diabetes and low birth weight: the role of paternal inheritance in the association of low birth weight and diabetes. Diabetes 49:445-449

22. Davey Smith G, Sterne JA, Tynelius P, Rasmussen F (2004) Birth characteristics of offspring and parental diabetes: evidence for the fetal insulin hypothesis. J Epidemiol Community Health 58:126-128

23. Lawlor DA, Davey Smith G, Ebrahim S (2002) Birth weight of offspring and insulin resistance in late adulthood: cross sectional survey. BMJ 325:359

24. Wannamethee SG, Lawlor DA, Whincup PH, Walker M, Ebrahim S, Davey-Smith G (2004) Birthweight of offspring and paternal insulin resistance and paternal diabetes in late adulthood: cross sectional survey. Diabetologia 47:12-18

25. McCarron P, Davey Smith G, Hattersley AT (2004) Type 2 diabetes in grandparents and birth weight in offspring and grandchildren in the ALSPAC study. J Epidemiol Community Health 58:517-522

26. Knight B, Shields BM, Hattersley AT (2006) The Exeter Family Study of Childhood Health (EFSOCH): study protocol and methodology. Paediatr Perinat Epidemiol 20:172-179

27. Townsend P, Phillimore P, Beattie A (1988) Health and deprivation: inequality and the North. Croom Helm, London

28. Matthews DR, Hosker JP, Rudenski AS, Naylor BA, Treacher DF, Turner RC (1985) Homeostasis model assessment: insulin resistance and beta-cell function from fasting plasma glucose and insulin concentrations in man. Diabetologia 28:412-419

29. Levy JC, Matthews DR, Hermans MP (1998) Correct homeostasis model assessment (HOMA) evaluation uses the computer program. Diabetes Care 21:2191-2192

30. Freeman JV, Cole TJ, Chinn S, Jones PR, White EM, Preece MA (1995) Cross sectional stature and weight reference curves for the UK, 1990. Arch Dis Child 73:17-24

31. Godfrey KM, Hales CN, Osmond C, Barker DJ, Taylor KP (1996) Relation of cord plasma concentrations of proinsulin, 32-33 split proinsulin, insulin and C-peptide to placental weight and the baby's size and proportions at birth. Early Hum Dev 46:129-140

32. Holte J, Bergh T, Berne C, Berglund L, Lithell H (1994) Enhanced early insulin response to glucose in relation to insulin resistance in women with polycystic ovary syndrome and normal glucose tolerance. J Clin Endocrinol Metab 78:1052-1058

33. Le Stunff C, Bougneres P (1994) Early changes in postprandial insulin secretion, not in insulin sensitivity, characterize juvenile obesity. Diabetes 43:696-702

34. Lillioja S, Nyomba BL, Saad MF et al (1991) Exaggerated early insulin release and insulin resistance in a diabetes-prone population: a metabolic comparison of Pima Indians and Caucasians. J Clin Endocrinol Metab 73:866-876

35. Wang HS, Lee JD, Soong YK (1995) Effects of labor on serum levels of insulin and insulin-like growth factor-binding proteins at the time of delivery. Acta Obstet Gynecol Scand 74:186-193

36. Lindsay RS, Walker JD, Halsall I et al (2003) Insulin and insulin propeptides at birth in offspring of diabetic mothers. J Clin Endocrinol Metab 88:1664-1671 